\title{
Comparative study of active infrared thermography, laser vibrometry and laser ultrasonic techniques in application to the inspection of graphite/epoxy composite parts
}

\author{
by V.P. Vavilov*, A.S. Karabutov**********, A.O. Chulkov*, D.A. Derusova*, \\ A. Moskovchenko*, E.B.Cherepetskaya ${ }^{* \star}$, E.A.Mironova*** \\ * Tomsk Polytechnic University, 634050, Tomsk, Russia, vavilov@tpu.ru \\ ** ILC M.V. Lomonosov Moscow State University, 119991, Moscow, Russia \\ *** NUST MISIS, 119049, Moscow, Russia \\ **** ILIT RAS - Branch of the FSRC «Crystallography and Photonics» RAS, 140700, Shatura, Russia
}

\begin{abstract}
Three nondestructive testing techniques, namely, active infrared thermography, laser vibrometry and laser ultrasound, have been comparatively applied in the inspection of a graphite epoxy sample characterized by a complicated geometry to demonstrate advantages and drawbacks of each technique in the detection of various types of defects.
\end{abstract}

\section{Introduction}

Most previous studies in nondestructive testing (NDT) of composite materials have been related to particular inspection techniques or particular types of defects in flat reference samples. In graphite epoxy composites, either impact damage or insert-like defects have been investigated [1-2]. Few works were dedicated to comparison of efficiency of various NDT methods applied to samples of complicated shape [3-4]. In this study, we apply three different NDT techniques, namely, active infrared (IR) thermography (with both optical and ultrasonic stimulation), laser vibrometry and laser ultrasound, to the detection of three types of defects (wall thinning, impact damage and cracks) in a curved part made of a graphite epoxy composite. Such parts are widely used in the aviation and aerospace industries.

\section{Test sample}

The $250 \times 100 \times 2.5 \mathrm{~mm}$ graphite epoxy test sample shown in figure 1 represented a fragment of a composite cylindrical case used in the aerospace industry. The main panel of a curved shape was strengthened with two types of ribs also made of graphite epoxy and contained three artificially-made defects (specified as 1, 2 and 3 in figure 1): two 12 mm-long thin vertical slits simulating cracks, local wall thinning with $15 \mathrm{~mm}$-diameter material loss from 40 to $50 \%$ and 15 $\mathrm{J}$ impact damage. Since thinning-like ("corrosion-type") defects are not typical in real composite parts, this kind of defect was used to show how local variations of main panel thickness and the presence of ribs affect surface temperature. Two other defects, in fact, simulated composite cracking that can occur in both lateral and in-depth directions. The throughthe-sample cracks could not be detected visually, therefore, to some extent, they simulated vertical "kissing" cracks which are typically hard to detect by applying common NDT techniques.

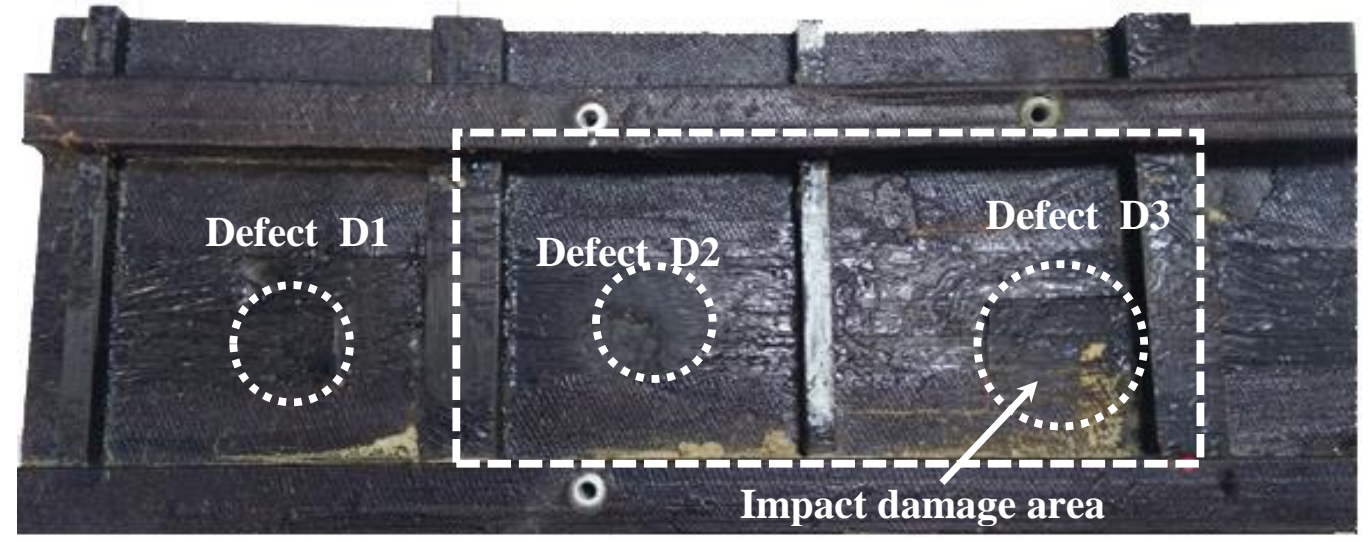

Fig. 1: Test graphite epoxy sample (rear view, defect 1 - through-the-sample("kissing") cracks, defect 2 - 40-50\% wall thinning, defect 3 - $15 \mathrm{~J}$ impact damage, square dashed area was tested by laser ultrasonics) 


\section{Inspection techniques and results}

\subsection{Optical stimulation}

The active IR thermographic method has been applied in its "classical" implementation, i.e. by involving optical and ultrasonic excitation. A flash tube $(5 \mathrm{~ms}, 1600 \mathrm{~J})$ and 2 halogen lamps (1 kw each) have been routinely used in a one-sided procedure to result in IR image sequences which included from 50 to 500 images. Both pulsed and thermal wave test procedures have been implemented. The laboratory setup has been designed around an Optris-450 IR module and included the home-made software providing data acquisition and processing by using most of known algorithms, merely to mention Fourier and wavelet transforms, principle component analysis (PCA), correlation, normalization, etc. An example of the resulting image obtained under optical stimulation is shown in figure $2 a$. The efficiency of each NDT technique (except laser ultrasound) has been evaluated by using the known concept of signal-to-noise ratio SNR $=\left|U_{d}-U_{n d}\right| / \sigma_{n d}$, where $U_{d, n d}$ are the signals in defect $(d)$ and non-defect $(n d)$ areas, and $\sigma_{n d}$ is the standard deviation in a chosen non-defect area.

Flash heating was not powerful enough to ensure a detectable temperature elevation over the area with material loss while two other defects appear clearly in the source image (figure 2a). Applying PCA has allowed to stress the material loss $(S N R=7)$ but worsened visibility of two other defects (figure $2 b)$. This is a typical selective feature of the PCA method which concentrates particular types of temperature patterns in particular components. The thermogram in Fig. 2c illustrates why halogen lamps are often used in active thermal NDT devices due to high energy delivered for some seconds of heating (see the results of the pulsed test in figure 2d). However, the highest SNR values for all three defects appeared in the case of low-frequency thermal waves (figure 2d).



a)

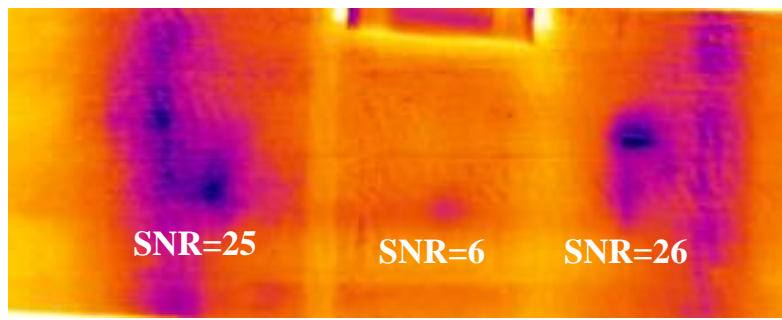

c)

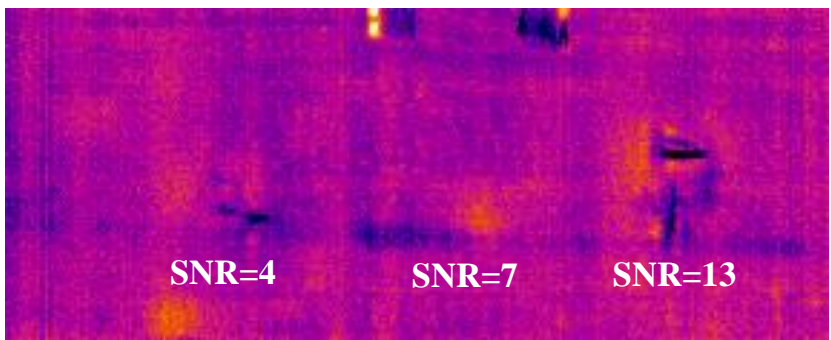

b)



d)

Fig. 2: Test results, optical heating: a-Xenon tube, source image, b-same as a), PCA image; c-halogen lamps, pulsed heating for $10 \mathrm{~s}$; PCA image; d-halogen lamps, frequency $0.07 \mathrm{~Hz}, P C A$ image

\subsection{Ultrasonic stimulation}

Ultrasonic stimulation was performed by using a magnetostrictive device providing continuous (long-pulsed) stimulation with $22 \mathrm{kHz}$ mechanical waves, while the electric power supplied to the magnetostrictive indentor was in the range from 50 to $500 \mathrm{~W}$ (see also [2-4]). When the indentor was firmly pressed onto the sample front surface in the sample centre, the defects 1 and 3 have been reliably detected providing fairly high SNR values while the indication of Defect 2 was hidden within the stimulation area (figure 3) it cannot detected by ultrasonic IR thermography because of a lack of internal friction. High SNR that are typical for this method of NDT values are conditioned by the principle of "dark field" that is characteristic for ultrasonic IR thermography. This means that defect-free areas do not change their temperature as a result of stimulation thus leading to very small values of temperature standard deviation $\sigma_{n d}$. The big divergence in the SNR values (see figure 3) are explained by a strong non-linear dependence of temperature signals on position of the simulation point. 


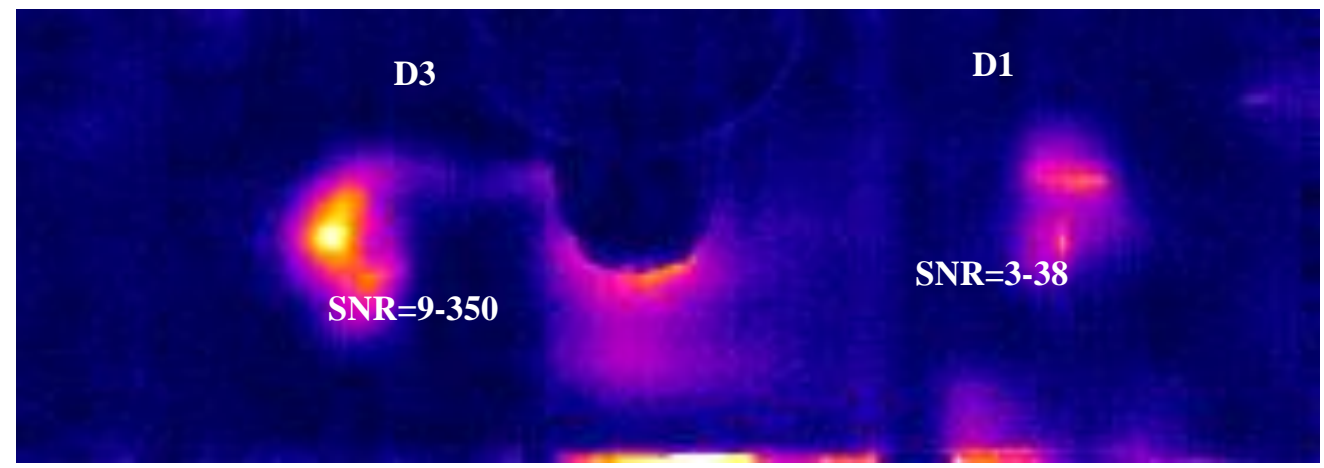

Fig. 3: Temperature distribution on sample surface under ultrasonic stimulation (22 $\mathrm{kHz}, 300 \mathrm{~W}$ electric power, magnetostrictive device in the centre)

\subsection{Laser vibrometry}

The technique of laser vibrometry combined low-power ultrasonic stimulation and vibration measurement by using a scanning laser Doppler vibrometer (SLDV) PSV-500-3D from Polytec (see also [5-7]). The input voltage up to $120 \mathrm{~V}$ amplitude from AWG-4163 function generator via a voltage amplifier AVA-1810 was applied to the piezotransducer clamped onto the front side of the sample to excite flexural waves in the frequency band from $25 \mathrm{~Hz}$ to 100 $\mathrm{kHz}$ with accuracy of $\pm 25 \mathrm{~Hz}$. A scanned area consisted of $20 \times 50$ points. The total vibration pattern shows the clearly visible defects under a relatively low ultrasonic load ( $\sim 50 \mathrm{~mW}$ of acoustic power). The magnitude of vibration velocities over defects varied from 0.7 to $1.5 \mathrm{~mm} / \mathrm{s}$ while amplitude of vibration velocity in defect-free areas is about $0.2 \mathrm{~mm} / \mathrm{s}$. In this way, the difference in velocities resulted in the values of signal-to-noise ratio of 11,5 and 12 for defects 1,2 and 3 respectively. It is worth noting that, when performing laser vibrometry, the inspection time reached 4 minutes if resonance frequencies adherent to the defects were not determined, while the search for particular resonances approximately doubled this time.

The most important information about defects is supplied by analyzing amplitude-frequency characteristics of samples under test. For each of three defects in the test sample from figure 1, we found resonance frequencies at which local vibrations are maximal (see figure 4). At the frequency of $2.275 \mathrm{kHz}$, the D1 area was characterized by mean vibration velocity of $62 \mu \mathrm{m} / \mathrm{s}$ and produced $S N R=23$ in regard to mean 'non-defect' vibration of $4.05 \mu \mathrm{m} / \mathrm{s}$ (see the formula for SNR above). In the D2 area, the intensity of resonance vibrations exceeded those in a non-defect area by 14 times reaching the value of $51 \mu \mathrm{m} / \mathrm{s}$ at the frequency of $3.25 \mathrm{kHz}$ : the corresponding SNR value was 21 . Respectively, in the D3 area, the mean vibration amplitude was $73 \mu \mathrm{m} / \mathrm{s}$ at the frequency of $2,65 \mathrm{kHz}$ (mean 'non-defect' value $6,5 \mu \mathrm{m} / \mathrm{s}$ ) with the maximum SNR=22.

Since the resonance frequencies for all defect areas were close, the technique of laser vibrometry allowed obtaining clear defect patterns in the image of total vibrations even if the corresponding SNR values were 2-4 times lower than in the case of resonance stimulation (figure 5 , wide frequency range).

We believe that the technique of resonance ultrasonic IR thermography allows determining defect location and dimensions by total vibration patterns for some minutes with a reasonable spatial resolution (few mm). However, a test time may become very long if, while determining local resonances, the number of spectral lines should be big enough to perform efficient Fourier transform and/or the number of scanned points should be increased. In this case, SNR values can be enhanced by 2-4 times (in a particular experiment) but the test time will be twice longer. In fact, a needed test time can be chosen by the operator depending on inspection task sand a required efficiency of defect evaluation.

\subsection{Laser ultrasonics}

Some limitations of this technique are relatively small scanned areas and low test productivity. Laser ultrasonic method uses broad-band ultrasonic waves generated by a laser pulse. The waves scattered by composite structural inhomogeneities are recorded with a high temporal resolution thus allowing depth resolution better than achieved in common ultrasonic NDT. 

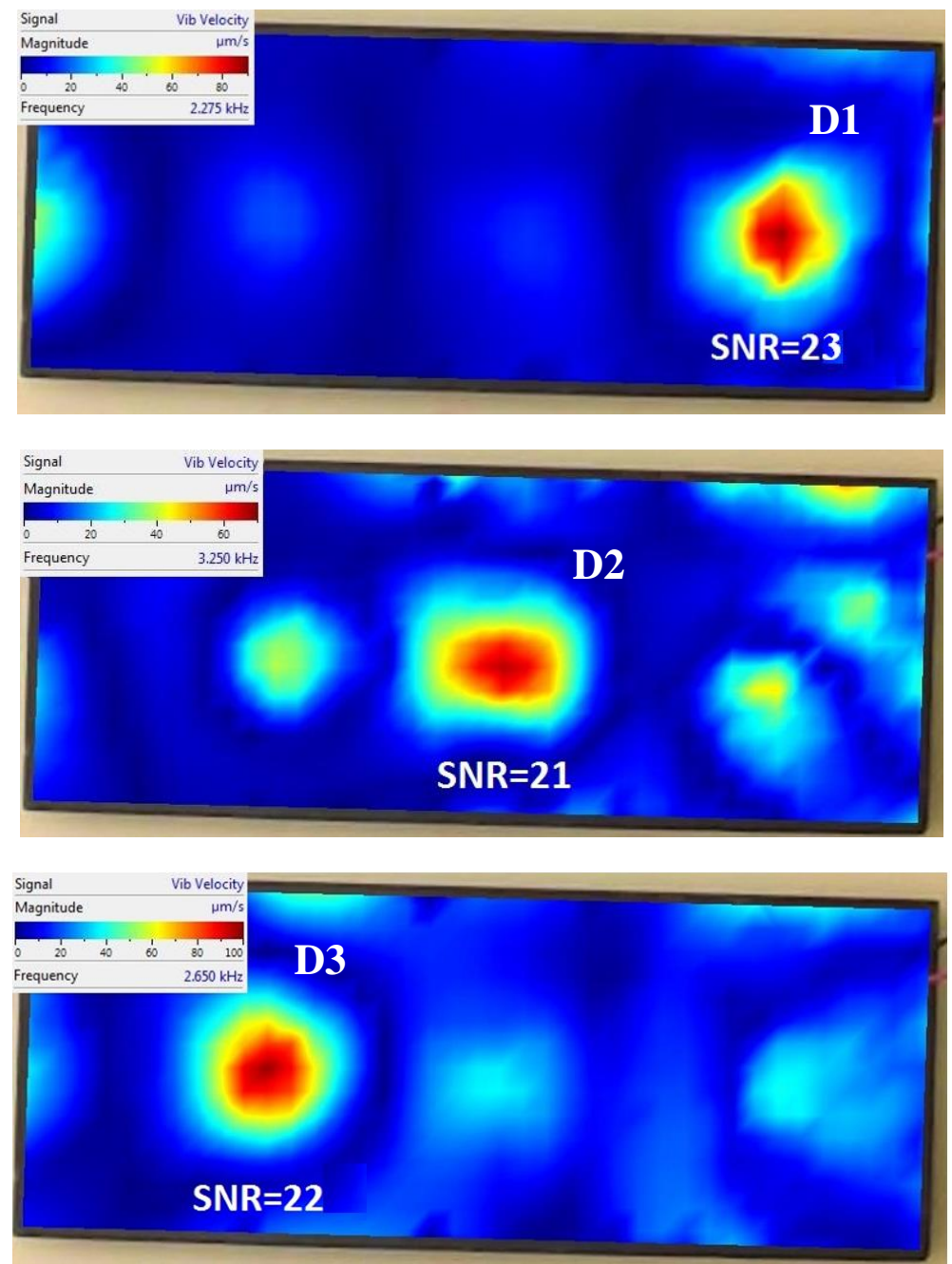

Fig. 4: Local resonance vibrations: D1 - $2.28 \mathrm{kHz}$ (top), D2 - $3.25 \mathrm{kHz}$ (middle), D3 - $2.65 \mathrm{kHz}$ (bottom)

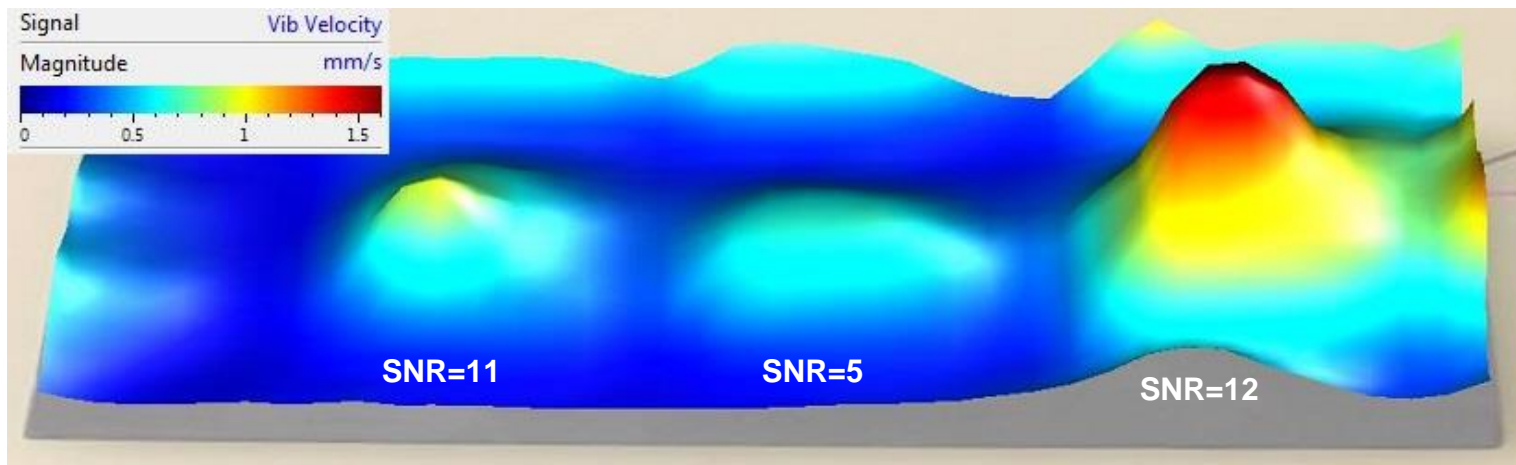

Fig. 5: Total vibration pattern obtained under low-power ultrasonic stimulation by applying SLDV technique (wide frequency range)

Figure 6 shows the interface of the computer program used in data processing. The size of the scanned area was $150 \times 80 \mathrm{~mm}$ (see figure 1), scanning step - $2 \mathrm{~mm}$. The signals obtained are represented in a 3D form (figure 6); the $X Y, X Z$ and $Y Z$ planes depict the sample cross-sections (slices) at fixed coordinates $Z, Y$ and $X$ respectively. The $X$ and $Y$ coordinates are fixed by the position of both the vertical and horizontal markers on the XY-plane, and the fixed Zcoordinate is determined by the horizontal marker on the $X Z$ plane. By correspondingly changing position of markers, it is possible to visualize the internal structure of the sample. The Z-coordinate corresponds to a time delay of the acoustic 
signal. In fact, by knowing velocity of ultrasonic waves in a test material, one can convert time delays in the in-depth coordinate Z, while grey tone images represent signal amplitudes.


Fig. 6: Example of laser ultrasonic test results (processing program interface)

In figure 6 , the sample front sample is represented by a white-colour line because here an ultrasonic pulse penetrates the composite from the medium of lower density; respectively, the rear surface appears as a wide dark line. It is clearly seen that the material is essentially inhomogeneous. It is also characteristic, that, in the defect D2 area, the bottom reflection of the ultrasonic waves occurs at the half sample thickness (see the XZ-plane). The area of impact damage (D3) contains plentiful cracks while the rear-surface signals are absent. In fact, acoustic signals cannot penetrate through the damaged area.

Thanks to high spatial resolution of the laser ultrasonics, results of applying this test method are sensitive to small variations in composite density thus producing images of which treatment is not straightforward requiring a considerable operators' experience. A figure of merit for laser ultrasonic images are local variations of acoustic impedance that is the product of the ultrasonic wave velocity and density. Therefore, areas with delaminations, disbonds, excessive porosity, etc. look "darker" in regard to areas of a regular texture. On the other hand, if the object texture is "rough", the contrast of defects can be relatively low. For example, fiber waviness makes the image of a lower contrast because, in the presented case, the slice thickness is about $15 \mu \mathrm{m}$, and the defect partially occupies other slices.

Figure 7 shows some sets of slices in the area of the defect D3. The XZ-planes correspond to different material depths: approximately $0.5,1.4$ and $2.9 \mathrm{~mm}$. By evaluating these results, one can see that the zone of internal structure damage has the form of an inverted cup with the cup bottom placed on the area of front surface impact. The cracks shown in the $X Y$ slices look like concentric circles of which radius increase with depth. 


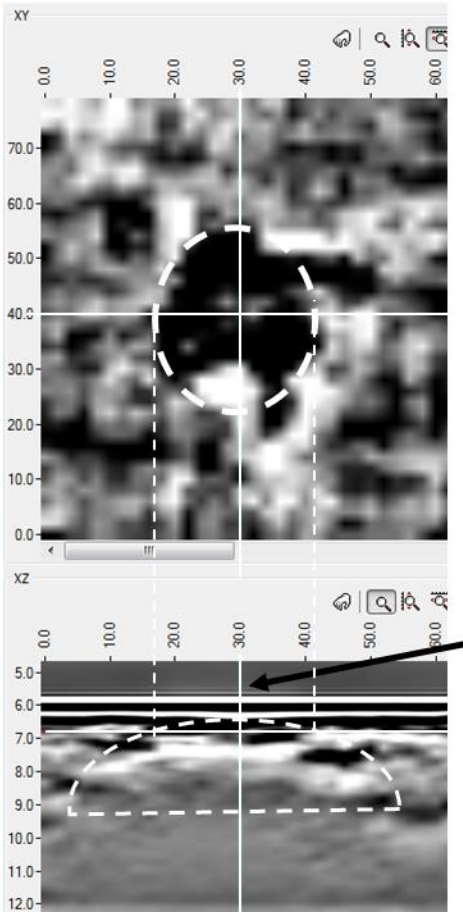

a)

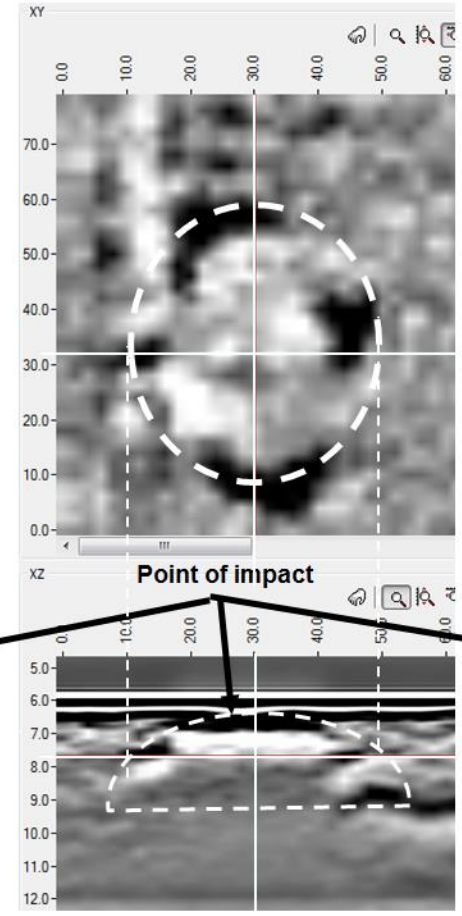

b)

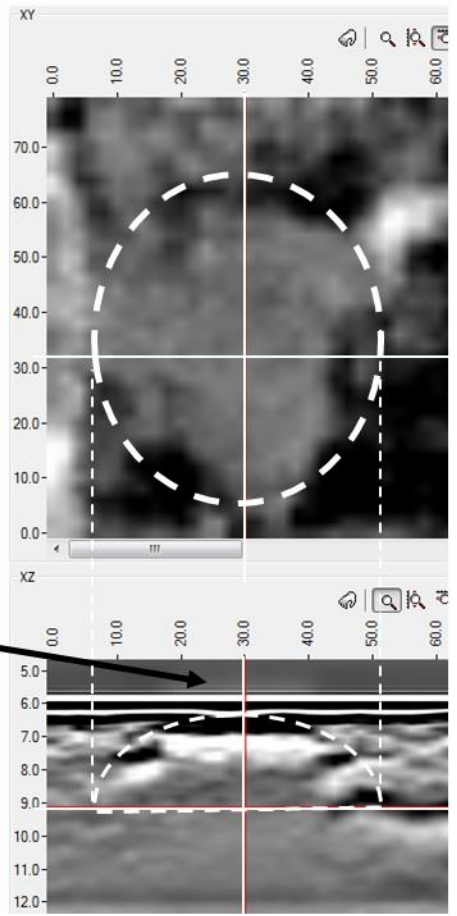

c)

Fig. 7: $X Z$ (top row) and $X Y$ (bottom row) slices: a - depth $0.5 \mathrm{~mm}, b-1.4 \mathrm{~mm}, \mathrm{c}-2.9 \mathrm{~mm}$

\section{Conclusion}

"Classical" IR thermographic NDT based on optical stimulation remains a reliable technique in the detection of various types of defects in graphite epoxy composites. It can be effectively complemented with ultrasonic stimulation which might be preferable if "kissing" defects are to be detected. Laser vibrometry combined with the principle of ultrasonic resonance stimulation appears as a very sensitive NDT method but it is still time-consuming and expensive. The technique of laser ultrasound seems to be a good addition to other NDT techniques due to its very high spatial resolution and tomographic data presentation. But this technique is also time-consuming and requires high operator qualification when identifying composite structural inhomogeneities.

This study was supported by the Russian Scientific Foundation grants \# 17-79-10143 (data processing) and \#17-19-01047 (experimental setups).

\section{REFERENCES}

[1] Klepka A., Pieczonka L., Staszewski W.J., Aymerich F., Impact Damage Detection in Laminated Composites by Non-Linear Vibro-Acoustic Wave Modulations. Composites Part B: Engineering. - Vol. 65, pp. 99-108, 2017.

[2] Vavilov V., Chulkov A., Derusova D., IR Thermographic Characterization Low Energy Impact Damage in Carbon/Carbon Composite by Applying Optical and Ultrasonic Stimulation. Proceedings of SPIE. - Vol. 9105, pp. 1-9. 2014.

[3] Rizi A.S., Hedayatrasa S., Maldague X., Vukhanh T., FEM Modeling of Ultrasonic Vibrothermography of a Damaged Plate and Qualitative Study of Heating Mechanisms. Infrared Physics and Technology. - Vol. 61, pp. 101-110. 2013.

[4] Solodov I., Bai J., Bekgulyan S., Busse G., A Local Defect Resonance to Enhance Acoustic Wave-Defect Interaction in Ultrasonic Nondestructive Testing. Applied Physics Letters. - Vol. 99, Article number 211911, pp. 109. 2011.

[5] Scruby C.B., Drain L.E. "Laser Ultrasonics: Techniques and Applications". Adam Hilger, Bristol, U.K., 1990.

[6] Podymova N.B., Karabutov A.A., Belyaev I.O. Broadband Laser-Ultrasonic Spectroscopy for Quantitative Evaluation of Porosity Effect on Acoustic Attenuation and Phase Velocity in CFRP Composites. - Proc. 11th Europ. Conf. NDT (ECNDT 2014), Oct. 6-10, 2014, Prague, Czech Republic, 10 p.

[7] Karabutov A.A., Kozhushko V.V., Pelivanov I.M., Podymova N.B. Nondestructive Evaluation of OneDimensional Periodic Structures by Transmission of Laser-Excited Wide-Band Acoustic Pulses. - Mech. Comp. Mater., Vol. 37, no. 2, pp. 153-158. 2001. 